\title{
Optimization of Hydrostatic Thrust Bearing Using Enhanced Grey Wolf Optimizer
}

\author{
İsmail ŞAHİ*, Murat DÖRTERLER**, Harun GÖKÇE*** \\ *Department of Industrial Design Engineering, Gazi University, 06500 - Ankara, Turkey, E-mail: isahin@gazi.edu.tr \\ **Department of Computer Engineering, Gazi University, 06500 - Ankara,Turkey, E-mail: dorterler@gazi.edu.tr \\ ***Defense Industries Research and Development Institute, TUBITAK, Ankara, Turkey, \\ E-mail: harungokce@yahoo.ca \\ crossref http://dx.doi.org/10.5755/j01.mech.25.6.22512
}

\section{Introduction}

Bearings are machine elements that allow movement in one or more directions between the two elements, with minimum friction, but hinder movement in the direction of force [1]. Many types of bearings are produced depending on the application areas and the desired function. They vary mainly in the direction of the load they carry, the type of rolling elements, or the type of bearing. The bearings are called hydrostatic if the fluids used in them are incompressible, and aerostatic, if compressible.

In hydrostatic bearings, pressure is applied to stabilize the external force and separate the surfaces from each other. The required pressure is supplied from the outside by means of a pump, and oil is sent to the bearings by this pressure. Bearings are widely used in industries requiring high accuracy (e.g. machine tools, measurement and control tools, process manufacturing and medical equipment) or in heavy-duty machines or at low-speed structures.

The need for precise mechanical and tribological properties of the hydrostatic bearings has made them an interesting study topic for optimisation studies. In recent years, swarm intelligence methods, which are nature-inspired algorithms, have been used extensively in the problem of optimization of hydrostatic thrust bearings [2-8]. The Grey Wolf Optimization (GWO) algorithm is a swarm intelligence method inspired by the hunting and leadership hierarchy method of grey wolfs [9]. Although GWO is a new method developed by Mirjalili et. al., it attracts the attention of researchers due to its successful performance in engineering optimisation problems [10-16].

In this study, the power-loss minimization problem of hydrostatic thrust bearing, which was firstly discussed by Siddall [2], was solved by using GWO. This problem is considered to be a very good benchmark problem due to the difficulty in the structure. 6 out of 7 constraints are active constraints considering an accuracy of 3 decimal places, and all the design variables are highly sensitive. The Accuracies of the Design variables are required from 9 to 15 decimal places [7]. In the study, the power-loss minimization problem of hydrostatic thrust bearing was applied to GWO for the first time. Previous studies compared their studies with the work of Siddall only and carried out performance analyses accordingly. Here, all previous studies on the subject were examined together for the first time and a comprehensive comparison was made. The most significant innovation of the study is the improvement on GWO's mathematical model. A new model (Enhanced GWO) which increases the range of solutions by keeping the search field wider is proposed.

\section{Literature review}

Bearing design is still an important subject for researchers, as the power of the pump has a direct impact on system parameters such as power, force, friction, and flow rate. The importance of hydrostatic deposits has increased with the development of application areas, and many studies have been carried out on this subject. The optimization studies on bearing geometry and surface roughness parameters, which significantly affect the performance of the bearings, are still a developing field of study today. The first hydrostatic bearings were developed by Slocum in 1995 to be used in high-pressure press machines [17]. In the developed system, the support equipment used for the bearing of the shaft had high strength and friction resistance. In 1996, Sinhasan and Sah investigated the system characteristics of bearing performance [18]. To determine the lubrication flow with different viscosities, solutions were obtained by using Reynolds equation, finite element method and optimization techniques. In another study conducted in 1996, an experimental study was conducted to evaluate the performance characteristics of the hydrostatic thrust bearing [19]. Performance characteristics of the system were confirmed measuring the oil film thickness, oil flow rate, pressure distribution and recess pressure.

The studies conducted to improve the performance of hydrostatic bearings show that the most important feature expected from the bearings should be a good abrasion resistance [20-25]. The thrust bearings are expected to have such properties as low friction coefficient, high abrasion resistance, high loading capacity, good erosion resistance, good thermal conductivity and low thermal expansion values in their selection. However, oil viscosity, oil film thickness, oil flow rate and pressure amount have significant effects on the performance of the system, as well.

Since the beginning of 2000, with the development of meta-heuristic optimization techniques and increasing computing capabilities, intelligent optimization studies on mechanical systems have accelerated. Optimization of hydrostatic deposits using meta-heuristic optimization techniques is a popular research topic. The aim of these studies is to minimize the loss of power during the operation of hydrostatic bearings [2-8].

The first study of the minimization of power loss during operation of hydrostatic bearings was carried out by 
Siddall [2]. The ADRANS method developed by Siddall using the Hooke and Jeeves (HJ) pattern search method resulted in a power reduction of 2,288.0 ft-lb/s (4.14 hp) in the existing bearings. Deb and Goyal solved the power minimization problem by using Genetic Adaptive Search (GeneAS) method based on Genetic Algorithm [3]. Their results were more successful than ADRANS method. In the same study, the results obtained with the Traditional Genetic Algorithm were shown, as well. The study showed that the bearing designed using GeneAS was much better than the other two techniques (BGA, HJ) and could withstand a higher film thickness. Solmaz et al. [4] performed single and multi-criteria optimization studies in circular hydrostatic bearings to ensure that the total power required for the bearing and the temperature increase in the bearing remained at minimum values. Multi-criteria optimization studies were shown to produce more effective results by applying sample methods. Coello's GA - Based approach proposes a multi-objective optimization technique instead of the penalty functions used in GA to overcome constraints [5]. The proposed method offers faster and better results than previous studies. In another study on the optimization of power loss, he suggested the Particle Swarm Optimization method [6]. He compared the previous studies with his study and he discussed the inconsistencies in the unit and design criteria with Siddall's study. Rao et al. applied the Teaching-Learning Optimization (TLBO) method to the Siddall's problem [7]. TLBO was shown to provide better results than other studies. Kentli and Sahbaz solved the problem by using a sequential quadratic programming (SQP) approach and compared their results with Siddall's study [8].

Another important research topic in hydrostatic bearings is the optimization of the load bearing capacities of the bearings. Fesanghary and Khonsari investigated the effect of cross-section and thickness variations of the film layer on hydrostatic deposits on load-bearing capacity of the bearings [26]. As a consequence of optimization with Sequential Quadratic Programming (SQP), different film thicknesses and the obtained forms were analyzed comparatively. As a result, SQP improved the load-bearing capacity of the bearing by over $90 \%$.

\section{The problem: optimization of hydrostatic thrust bear- ing design}

The problem of the optimization of hydrostatic thrust bearing was first defined by Siddall [2]. The aim of the optimization is to minimize the power loss during the operation of a hydrostatic plain bearing that is subjected to an axial load during operation (Fig. 1).

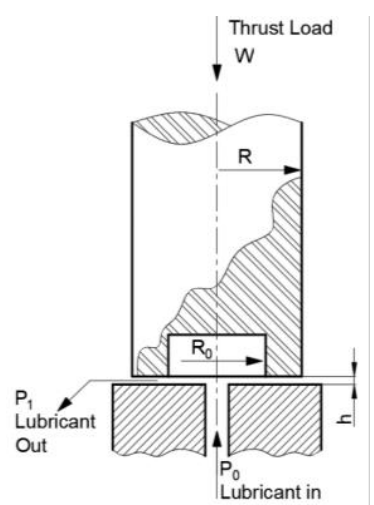

Fig. 1 Hydrostatic thrust bearing
Different methods were applied to the optimization problem by different researchers [3-8]. The following are the variables of the optimization, including four design variables and seven non-linear delimiters [2]: Flow rate $Q$, recess Radius $R_{0}$, Bearing step Radius $R$ and viscosity $\mu$.

The purpose function for minimizing the power loss of the hydrostatic thrust bearing is given in equation (1) [2]. Minimize:

$$
F(x)=\frac{Q P_{0}}{0.7}+E_{f}
$$

Seven non-linear constraints were identified in Eqs. (2-10) for the optimization [2,6].

Subject to:

weight capacity must be greater than weight of generator:

$$
G_{1}(x)=W-W_{s} \geq 0,
$$

where: $W$ is the weight capacity, which can be defined by the following equation:

$$
W=\frac{\pi P_{0}}{2} \frac{R^{2}-R_{0}^{2}}{\ln \frac{R}{R_{0}}} .
$$

Inlet oil-pressure required less than maximum pressure $P_{\max }$ available:

$$
G_{2}(x)=P_{\max }-P_{0} \geq 0,
$$

where: $P_{0}$ is the inlet pressure, which can be identified as:

$$
P_{0}=\frac{6 \mu Q}{\pi h^{3}} \ln \frac{R}{R_{0}} \text {. }
$$

fied:

Oil temperature rise should be less than that speci-

$$
G_{3}(x)=\Delta T_{\max }-T_{0} \geq 0 .
$$
film thickness:

Oil film thickness should be greater than minimum

$$
G_{4}(x)=h-h_{\text {min }} \geq 0 .
$$

Step radius must be greater than recess radius:

$$
G_{5}(x)=R-R_{0} \geq 0 \text {. }
$$

The six constraint limits on significance off exit loss:

$G_{6}(x)=0.001-\frac{\gamma}{g P_{0}}\left(\frac{Q}{2 \pi R h}\right) \geq 0$.

The seven constraint limits on contact pressure:

$G_{7}(x)=5000-\frac{W}{\pi\left(R^{2}-R_{0}^{2}\right)} \geq 0$, 
where: $E_{f}$ is the friction loss and is defined by the following equation:

$$
E_{f}=9336 Q \gamma C \Delta T \text {. }
$$

In the optimization, $\gamma$ (the weight density of oil) and $C$ (specific heat of oil) values given in equation 11, are defined as $0.0307 \mathrm{lb} / \mathrm{in}^{3}$ and $0.5 \mathrm{Btu} / \mathrm{lb} \mathrm{b}_{0} F$ respectively. $T$ (the temperature) given in Eq. (11) is defined by Eq. (12):

$$
\Delta T=2\left(10^{P}-560\right)
$$

where: $P$ is defined by the equation given below:

$$
P=\frac{\log _{10} \log _{10}(8.122 e 6 \mu+0.8)-C_{1}}{n} .
$$

In Eq. (13), $n$ and $C_{1}$ are one of the oil constants given in Table 1. Table 1 shows the values of $n$ and $C_{1}$ in different grades of oil. Selected in the study are $C_{1}=10.04$ and $n=-3.55$ for SAE 20 grade oil.

Table 1

Values of $n$ and $C_{1}$ for various grades of oil

\begin{tabular}{|c|c|c|}
\hline Oil & $C_{1}$ & $n$ \\
\hline SAE 5 & 10.85 & -3.91 \\
\hline SAE 10 & 10.45 & -3.72 \\
\hline SAE 20 & 10.04 & -3.55 \\
\hline SAE 30 & 9.88 & -3.48 \\
\hline SAE 40 & 9.83 & -3.46 \\
\hline SAE 50 & 9.82 & -3.44 \\
\hline
\end{tabular}

The film thickness $h$ can be calculated from the friction loss $E_{f}$ through the equation given in Eq. (14).

$$
h=\left(\frac{2 \pi N}{60}\right)^{2} \frac{2 \pi \mu}{E_{f}}\left(\frac{R^{4}}{4}-\frac{R_{0}^{4}}{4}\right) .
$$
as follows:

Other specifications of the design optimization are Ws $($ Weight of generator $)=101000 \mathrm{lb}(45804.99 \mathrm{~kg}) ; P_{\max }$ $($ maximum pressure available $)=1000$ psi $(6.89655 \times 106$ $\mathrm{Pa}) ; \Delta T_{\max }$ (maximum temperature rise $)=50^{\circ} \mathrm{F}\left(10^{\circ} \mathrm{C}\right) ; h_{\min }$ (minimum oil thickness $)=0.001$ in. $(0.00254 \mathrm{~cm}) ; g=32.3$ $\times 12=386.4 \mathrm{in} . / \mathrm{seg}^{2}\left(981.465 \mathrm{~cm} / \mathrm{seg}^{2}\right)$ and $\mathrm{N}$ (angular speed of shaft $)=750$ RPM.

The ranges of values that the design variables of the optimization could have are as follows:

$1.000 \leq R \leq 16.000$

$1.000 \leq R O \leq 16.000$

$1.0 \times 10^{-6} \leq \mu \leq 16 \times 10^{-6}$

$1.000 \leq Q \leq 16.000$.

\section{Grey Wolf Optimizer}

The Grey Wolf Optimization (GWO) algorithm is population-based and an intuitive method inspired by the hunting method of wolves in nature [9]. The method is based on the rigid hierarchy of grey wolves and their hunting behaviour. Each individual in the herd undertakes one of the alpha, beta, delta and omega roles defined from top to the bottom. Alpha is the leader of the herd, and he makes the decisions. Beta acts as a consultant of the alpha wolf. He has an auxiliary role in the activities of the herd and the decisions of the alpha. Delta wolves are dominant against Omega while obeying alpha and beta. In the herd, scouts, sentinels, elders, hunters, and caretakers are included in the delta group, and each has his own defined responsibilities. Omega wolf is at the bottom of the hierarchy. Omega, who has to obey individuals from the upper levels of the hierarchy, seems to be not an important individual in the herd, but has a role in preventing internal conflicts and problems.

\subsection{Mathematical model of the GWO}

In GWO, each solution in the population corresponds to a wolf in the herd. Alpha $\alpha$ is considered as the most suitable solution. The second and third best solutions are assumed to be Beta $\beta$ and Delta $\delta$, respectively. Omega $\omega$ is considered as the remaining solutions.. The hunting area also represents the search field, and the hunt represents the optimal solution.

In the hunting, primarily grey wolves encircle the hunt. The mathematical model of GWO developed by Mirjalili et. al is given Equation 15-25 [9]. The mathematical model of herd encircling behaviour was defined as in Eqs. (15) and (16). In each iteration, the position of each individual in the herd is updated by using Eqs. (15) and (16).

$$
\begin{aligned}
& \vec{D}=\left|\vec{C} \cdot \vec{X}_{p}(t)-\vec{X}(t)\right|, \\
& \vec{X}(t+1)=\vec{X}_{p}(t)-\vec{A} \cdot \vec{D} .
\end{aligned}
$$

In Eq. (16), t represents the current iteration, $\vec{A}$ and $\vec{C}$ the coefficient factors, and $\overrightarrow{X_{p}}$ position vector of the hunt, and $\vec{X}$ of the wolf. $\vec{A}$ and $\vec{C}$ coefficient factors are calculated with the help of Eqs. (17) and (18), respectively:

$$
\begin{aligned}
& \vec{A}=2 \vec{a} \cdot \vec{r}_{1}-\vec{a}_{1}, \\
& \vec{C}=2 \cdot \vec{r}_{2},
\end{aligned}
$$

where: $\vec{a}$ decreases linearly from 2 to 0 during iteration. $\vec{r}_{1}$ and $\vec{r}_{2}$ vectors, however, are the vectors having random values in the range of $[0,1] . \vec{C}$ and $\vec{A}$ factors are effective in finding the position of the hunt in search field. $\vec{C}$ defines the weight of the position of the hunt and is used for the calculation of $\vec{D}$, the position of the individual in the herd. $\vec{A}$ represents the condition of the wolf getting closer to or moving away from the hunt. If $|A|>1$, the wolf is getting away from the hunt, if $|A|<1$, it is getting closer to the hunt.

Grey wolves have the ability to recognize the location of the hunt and to encircle them. The hunting is usually guided by the alpha. Beta and delta may occasionally participate in occasional hunting. To mathematically simulate the hunting behaviour of grey wolves in GWO, alpha (best candidate solution), beta and delta are assumed to have a better knowledge of the potential location of the hunt. Therefore, the first three best solutions obtained during the algorithm are stored and the locations of other search agents (omegas) are updated according to the location of the best search agents (Eqs. (19-25)). 


$$
\begin{aligned}
& \vec{D}_{\alpha}=\left|\vec{C}_{1} \cdot \vec{X}_{\alpha}-\vec{X}\right|, \\
& \vec{D}_{\beta}=\left|\vec{C}_{2} \cdot \vec{X}_{\beta}-\vec{X}\right|, \\
& \vec{D}_{\delta}=\left|\vec{C}_{3} \cdot \vec{X}_{\delta}-\vec{X}\right|, \\
& \overrightarrow{X_{1}}=\overrightarrow{X_{\alpha}}-\overrightarrow{A_{1}} \cdot\left(\overrightarrow{D_{\alpha}}\right), \\
& \left.\overrightarrow{X_{2}}=\overrightarrow{X_{\beta}}-\overrightarrow{A_{2}} \cdot \overrightarrow{\left(D_{\beta}\right.}\right), \\
& \left.\overrightarrow{X_{3}}=\overrightarrow{X_{\delta}}-\overrightarrow{A_{3}} \cdot \overrightarrow{\left(D_{\delta}\right.}\right), \\
& \vec{X}(t+1)=\frac{\overrightarrow{X_{1}}+\overrightarrow{X_{2}}+\overrightarrow{X_{3}}}{3} .
\end{aligned}
$$

\subsection{Enhanced GWO}

In the experiments, it was observed that the performance of GWO in the solution of the problem was low compared to the results of other studies in the literature. In order to achieve better results, the change was made in the value of the $a$ in Eq. 26. The $a$ value is of critical importance in

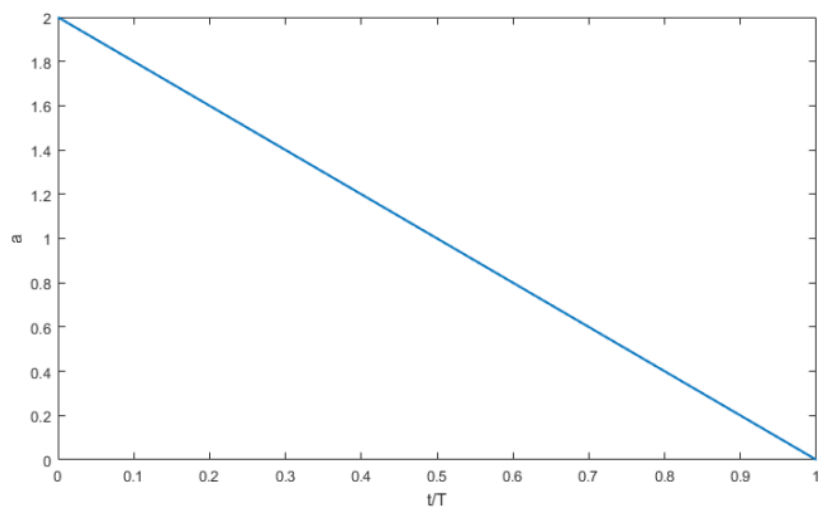

a
GWO (Fig. 3). As shown in Fig. 2, a, according to equation (26), the $a$ value decreases linearly from 2 to 0 during the search process, and the search area gets narrowed accordingly. In view of the difficulties in providing the constraints in the structure of the problem and the sensitivity of the design variables, it is preferred to use the $a^{\prime}$ value defined in Eq. (27) instead of the $a$ value in the mathematical model.

$$
\begin{aligned}
& a=2-2 \times \frac{t}{T}, \\
& a^{\prime}=\left\{\begin{array}{l}
2-4\left(\frac{t / T}{T}\right)^{2}, 0 \leq t / T \leq T / 2 \\
4\left(\frac{t / T-T}{T}\right)^{2}, T / 2 \leq t / T \leq T
\end{array}\right\} .
\end{aligned}
$$

As shown in Fig. 2, b, the $a^{\prime}$ value draws a splinebased curve in the search process from 2 to 0 . With the use of the $a^{\prime}$ value ; it was aimed to make more iterations around the best solution in the second half while increasing the variety of the current solutions by keeping the search area wider in the first half of the search process. This allows the algorithm to achieve better results

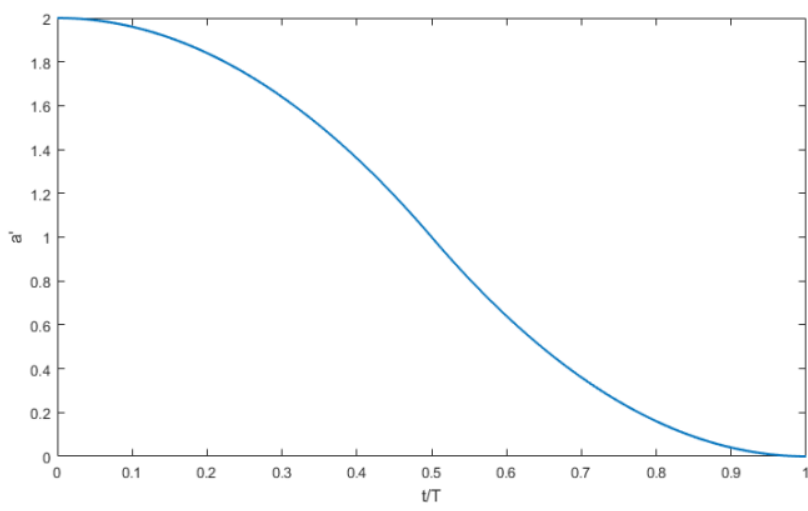

b

Fig. 2 The chart of the $a$ value during the iteration: a - the original version, $\mathrm{b}$ - the modified version

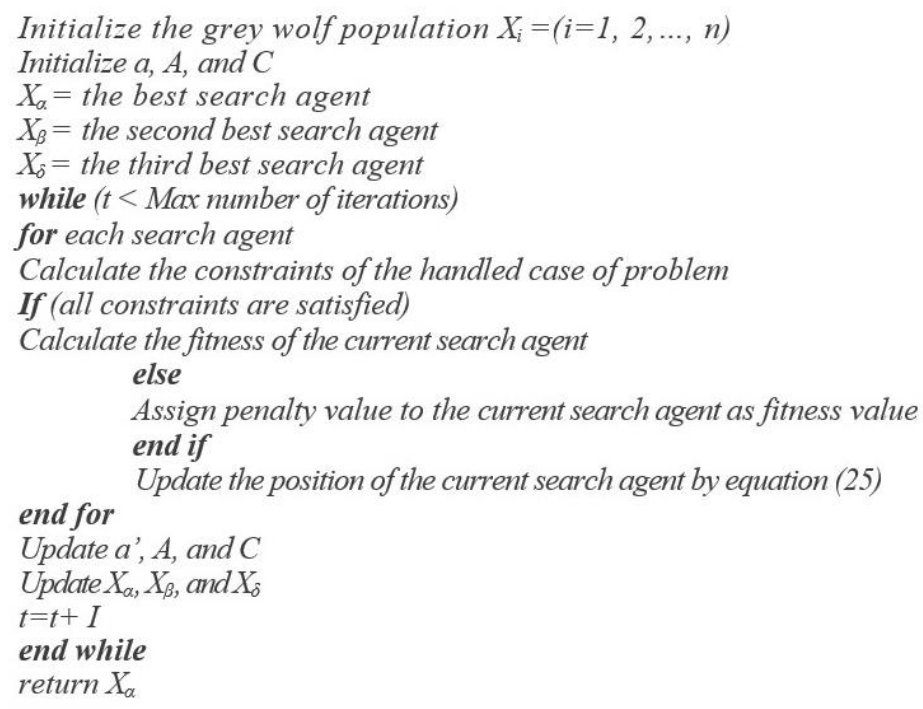




\section{Results and discussion}

The present study aims to minimize the power loss of hydrostatic bearings formulated by Siddall [2]. For the solution, Siddall used the ADRANS method based on Hooke and Jeeves $(\mathrm{HJ})$ pattern search method. In this study, the performance of GWO and EGWO in solving the problem by using the objective function, design constraints, variables and parameters defined by Siddall was tested. In addition, the performances of both methods were compared with the performance of the GA, GeneAS, PSO, TLBO and Gene-SQP methods applied to the problem in previous studies.

For the solution of the problem through GWO and EGWO, the codes developed by Mirjalili were employed [9]. In the existing codes, necessary modifications were made by considering the objective function along with design variable ranges and constraints for both cases.

The statistical performance of GWO and EGWO was evaluated in the first stage of the experimental studies. For this purpose, the population size for both algorithms was 50 , and the number of iterations was 500. Performance studies were conducted on MATLAB 2016b software on a Windows 10 x64 machine with Intel (R) Core (TM) i7 $3.3 \mathrm{GHz}$
CPU and 8 GB of RAM. The best, the worst, average, success percentage (SP) and standard deviation (SD) values of the obtained results were calculated by running each algorithm 100 times. SP shows the stability of the algorithm by evaluating the results obtained with the algorithms running a different number of times. In this context, the global optimum $(\mathrm{F} *)$ value should be less than $0.1 \%$ of the difference between the best obtained $(\mathrm{F})$ value and the global optimum value obtained for the successful acceptance of the result (Eq. (28)).

$$
\left|F^{*}-F / F^{*}\right|<1 E-3 \text {. }
$$

The performances of GWO and EGWO indicate that the results of EGWO are superior to GWO in every perspective (Table 2).

The statistical results of GWO and EGWO were also compared with those of Rao et. al. [7]. Rao et. al. applied TLBO and ABC algorithm to the problem. Table 3 shows that EGWO has a more stable performance in SP mean and worst values than that of Rao et. al. In the best value comparison, the difference is negligible even if it is left behind compared to TLBO and ABC.

Table 2

Statistical comparison of EGWO and GWO

\begin{tabular}{|l|l|l|l|l|l|}
\hline & Best $F_{x}$ & Worst $F_{x}$ & Mean $F_{x}$ & $S D$ & $S P$ \\
\hline GWO & 19531.65245856190 & 19982.44510141746 & 19613.23633162136 & 88.600667108268382 & 0.00 \\
\hline EGWO & 19505.57612078090 & 19808.38496195806 & 19532.93037771405 & 45.786563676010495 & 0.64 \\
\hline
\end{tabular}

Table 3

Statistical comparison of EGWO, GWO, TLBO, ABC

\begin{tabular}{|l|l|l|l|l|}
\hline & Best $F_{x}$ & Mean $F_{x}$ & Worst $F_{x}$ & $S P$ \\
\hline TLBO[8] & 19505.316 & 21572.49576 & 25161.61524 & 0.19 \\
\hline ABC [8] & 19505.31312 & 22338.648 & 25738.032 & 0.05 \\
\hline GWO & 19531.65245856190 & 19613.23633162136 & 19982.44510141746 & 0.00 \\
\hline EGWO & 19505.57612078090 & 19532.93037771405 & 19808.38496195806 & 0.64 \\
\hline
\end{tabular}

In Fig. 4, convergence rates of the algorithms are presented. Fig. 4, a shows the convergence curves of GWO and EGWO. Fig. 4, b is taken exactly from the study of Rao et al. For the compatibility of the curves, function values were calculated in units of $\mathrm{ft} \mathrm{lb} / \mathrm{s}$. As shown in Fig. 4, a,

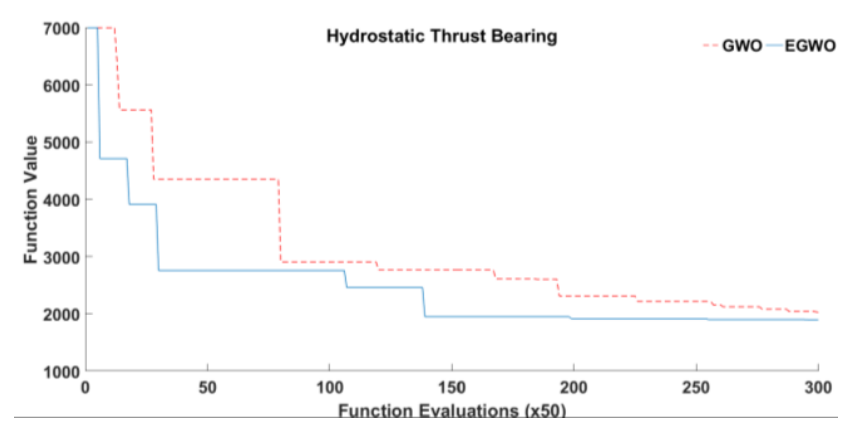

a
EGWO converges faster than GWO and makes more searches around the optimum solution. The convergence of EGWO is similar to that of TLBO and ABC whereas GWO's convergence lags behind others. These results indicate that the change in GWO results are appropriate for the purpose.

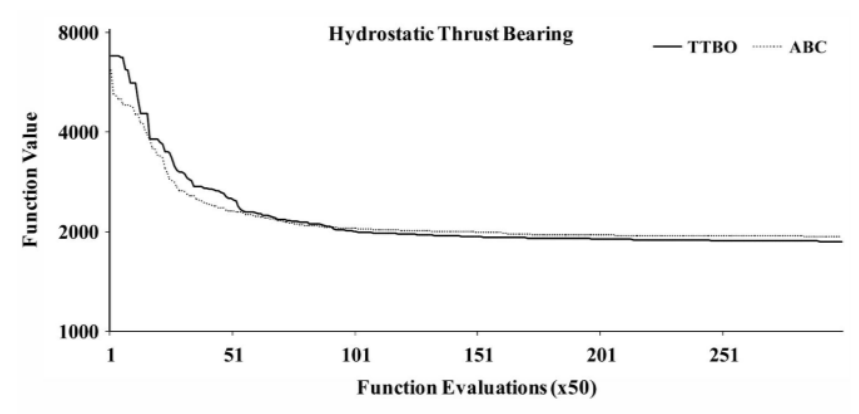

b

Fig. 4 The Coverage rate of the algorithms for the problem: a - the coverage rate of GWO and EGWO; $b$ - the coverage rate of TLBO and $\mathrm{ABC}$ [7]

The obtained optimum values in Table 4 are given in comparison with previous studies. Minor differences were observed in the units and design definitions in the previous studies. In Siddall's book, the fourth constraint (g4) and the sixth one (g6) are multiplied by 108 , and the fifth constraint and the third one are multiplied by 105 and 2000, respectively. The unit of fitness value from Deb [3] and Coello [5] used 560.0 while Siddall used 559.7 (Eq. (12)).

In Siddall's book, the fourth constraint (g4) and the sixth one (g6) are multiplied by 108 , and the fifth con- 


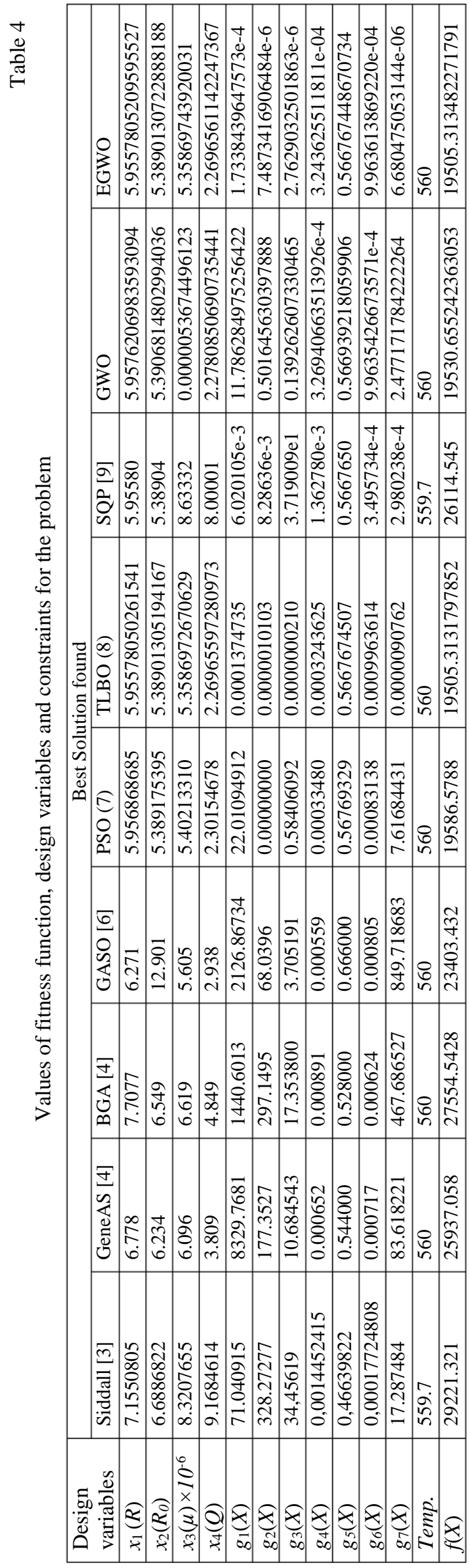

straint and the third one are multiplied by 105 and 2000, respectively. The unit of fitness value from Deb and Coello's papers is foot-pounds per second while Siddall used inchespounds per second. In order to facilitate the comparison, the optimum values presented in previous studies were arranged by Siddall's definition and transferred to Table 4. EGWO can reach the best-known value, but GWO does not exhibit the same success (Table 4).

\section{Conclusion}

In this study, GWO was applied for the first time to solve the design problem of minimum power loss of hydrostatic thrust bearing. Moreover, enhanced GWO (EGWO) was proposed due to the lower performance of the GWO on the problem. The performance of the both of them were compared with the previous studies. The results show that GWO is not capable of solving the problem as much as previous studies. On the other hand, EGWO got the know best fitness value with highest success rate.

The consistency and statistical performance of the EGWO show that this method can be used in the optimization of machine elements. In the future, we are planning to investigate the performance of the proposed method on the well-known engineering optimization problems.

\section{Disclosure Statement} authors.

No potential conflict of interest was reported by the

\section{References}

1. Achen, P.; Brink, T.; Vael, G. 2010. A robust hydrostatic thrust bearing for hydrostatic machines, 7th International Fluid Power Conferences, Aachen, Germany, Mar.

2. Siddall, J. N. 1982. Optimal Engineering Design, Marcel Dekker, New York, USA, ISBN 978-0824716332.

3. Deb, K.; Goyal, M. 1995. Optimizing engineering design using a Combined Genetic Search, Proc. of the Seventh International Conference on Genetic Algorithms, Pittsburgh, USA, July 1995, Morgan Kaufmann Publishers Inc. San Francisco, CA, USA.

4. Solmaz, E.; Babalık, F.C.; Öztürk, F. 2002. Optimization studies according to multi-criteria based design in circular thrust bearings, Uludağ University Journal of the Faculty of Engineering 7(1):57-70. https://doi.org/10.17482/uujfe.47735.

5. Coello, C. A. 2000. Theoretical and numerical constraint-handling techniques used with evolutionary algorithms, Engineering Optimization 32(3): 275-308 https://doi.org/10.1016/S0045-7825(01)00323-1.

6. He, S.; Prempain, E.; Wu, Q. H. 2004. An improved Particle Swarm Optimizer for mechanical design optimization problems, Engineering Optimization 36(5):585605. https://doi.org/10.1080/03052150410001704854.

7. Rao, R. V.; Savsani, V. J.; Vakharia, D. P. 2011. Teaching-learning-based optimization: A novel method for constrained mechanical design optimization problems, Computer-Aided Design 43(3):303-315. https://doi.org/10.1016/j.cad.2010.12.015. 
8. Kentli, A.; Sahbaz, M. 2014. Optimization of hydrostatic thrust bearing using Sequential Quadratic Programming, Oxidation Communications 37(4): 11441152.

9. Mirjalili, S.; Mirjalili, S. M.; Lewis, A. 2014. Grey wolf optimizer, Advances in Engineering Software 69: 46-61. https://doi.org/10.1016/j.advengsoft.2013.12.007.

10. Mirjalili, S.; Saremia, S.; Mirjalili, S. M.; Coelho, L. S. 2016. Multi-objective grey wolf optimizer: A novel algorithm for multi-criterion optimizations, Expert System with Application 47(1):106-119. https://doi.org/10.1016/j.eswa.2015.10.039.

11. Dörterler, M.; Şahin, İ.; Gökçe, H. 2019. A grey wolf optimizer approach for optimal weight design problem of the spur gear, Engineering Optimization 51(6): 10131027. https://doi.org/10.1080/0305215X.2018.1509963.

12. Sulaiman, M. H.; Mustaffab, Z.; Mohameda, M. R.; Alimana, O. 2015. Using the grey wolf optimizer for solving optimal reactive power dispatch problem, Applied Soft Computing 32: 286-292. https://doi.org/10.1016/j.asoc.2015.03.041.

13. Yildiz, B. S.; Yildiz, A. R. 2018. Comparison of grey wolf, whale, water cycle, ant lion and sine-cosine algorithms for the optimization of a vehicle engine connecting rod, Materials Testing 60: 311-315.

https://doi.org/10.3139/120.111153.

14. Wen, L.; Jianjun, J.; Ximing, L.; Mingzhu, T. 2018. An exploration-enhanced grey wolf optimizer to solve high-dimensional numerical optimization, Engineering Applications of Artificial Intelligence 68: 63-80. https://doi.org 10.1016/j.engappai.2017.10.024.

15. Zhang, S.; Zhou, Y.; Li, Z.; Pan, W. 2016. Grey wolf optimizer for Uunmanned combat aerial vehicle path planning, Advances in Engineering Software, 99: 121136. https://doi.org/10.1016/j.advengsoft.2016.05.015.

16. Sahin, İ.; Dörterler, M.; Gökçe, H. 2017. Optimum Design of Compression Spring According to Minimum Volume Using Grey Wolf Optimization Method, Gazi Journal of Engineering Sciences 3(2)2: 21-27.

17. Kane, N. R.; Sihler, J.; Slocum, A. H. 2003. A hydrostatic rotary bearing with angled surface self-compensation, Precision Engineering 27: 1 - 15. https://doi.org/10.1016/S0141-6359(02)00194-0.

18. Sinhasan, R.; Sah, P. L. 1996. Static and dynamic performance characteristics of an orifice compensated hydrostatic journal bearing with non-newtonian lubricants, Tribology International 29, 6: 515-526. https://doi.org/10.1016/0301-679X(95)00115-K.

19. Osman, T. A.; Dorid, M.; Safar, Z. S.; Mokhtar, M. O. A. 2006. Experimental assessment of hydrostatic thrust bearing performance, Tribology International 29(3): 233-239. https://doi.org/10.1016/0301-679X(95)00078-I.

20. Peeken, H. 1975. Die Berechnung Hydrostatischer Lager", Springer Verlag, Berichte.

21. Yuan, S.; Zhou, D. 1997. Design procedure of an advanced spherical hydrostatic bearing used in rotary forging presses, International Journal Machine Tools Manufacturing 37(5): 649-656. https://doi.org/10.1016/S0890-6955(96)00003-X.
22. Elsharkawy, S. A.; Guedouar, L. H. 2001. An inverse solution for finite journal bearings lubricated with couple stress fluids, Tribology International 34(2): 107-118. https://doi.org/10.1016/S0301-679X(00)00145-6.

23. HongXin, S.; JianPing, Z.; QiBo, P.; HaiYang, L.; JiuTia, L. 2012. Multi-objective interplanetary trajectory optimization combining low-thrust propulsion and gravity-assist maneuvers, Sci. China Technol. Sci. 55(3): 841-847. https://doi.org/10.1007/s11431-011-4705-5.

24. Pavlioglou, S.; Mastrokalos, M. E.; Papadopoulos, C.; Kaiktsis, L. 2015. Tribological optimization of thrust bearings operated with lubricants of spatially varying viscosity, Journal of Engineering for Gas Turbines and Power 137(2): 1-11.

https://doi.org/10.1115/1.4028371.

25. Solmaz, E. 2014. Optimization studies in circular thrust bearings, Uludağ University Journal of the Faculty of Engineering 9: 1-9.

26. Fesanghary, M.; Khonsari, M.M. 2012. Topological and shape optimization of thrust bearings for enhanced load-carrying capacity, Tribology International 53: 1221.

https://doi.org/10.1016/j.triboint.2012.03.018.

\section{İ. Şahin, M. Dörterler, H. Gökçe \\ OPTIMIZATION of HYDROSTATIC THRUST \\ BEARING USING ENHANCED GREY WOLF OPTIMIZER}

S u m m a r y

The need for precise mechanical and tribological properties of the hydrostatic bearings has made them an interesting study topic for optimisation studies. In this paper, power-loss minimization problems of hydrostatic thrust bearings were solved through Grey Wolf Optimizer (GWO). Grey Wolf Optimizer is a meta-heuristic optimization method standing out with its successful applications in engineering design problems. Power-loss minimization problem of hydrostatic thrust bearings was applied on Grey Wolf Optimizer (GWO) for the first time. The obtained results were evaluated together with the previous studies conducted, and a detailed comparison was made. The most significant innovation of the study is the innovation made in the mathematical model of the GWO. A new model (Enhanced GWO, EGWO) that increases the variety of valid solutions was proposed. The comparisons made both with GWO and other studies in the literature show that EGWO got the best known fitness value with the highest success rate. The consistency and statistical performance of the EGWO show that this method can be used in the optimization of machine elements.

Keywords: Hydrostatic Thrust Bearing, Grey wolf Optimiser, meta-heuristic, Enhanced GWO, engineering optimisation.

Received January 16, 2019

Accepted October 14, 2019 